\title{
FORMACIÓN DEL SUJETO QUE NECESITA LA SOCIEDAD CONTEMPORÁNEA \\ EL MACROCURRÍCULO COMO UNA EXPERIENCIA DE PENSAMIENTO COMPLEJO
}

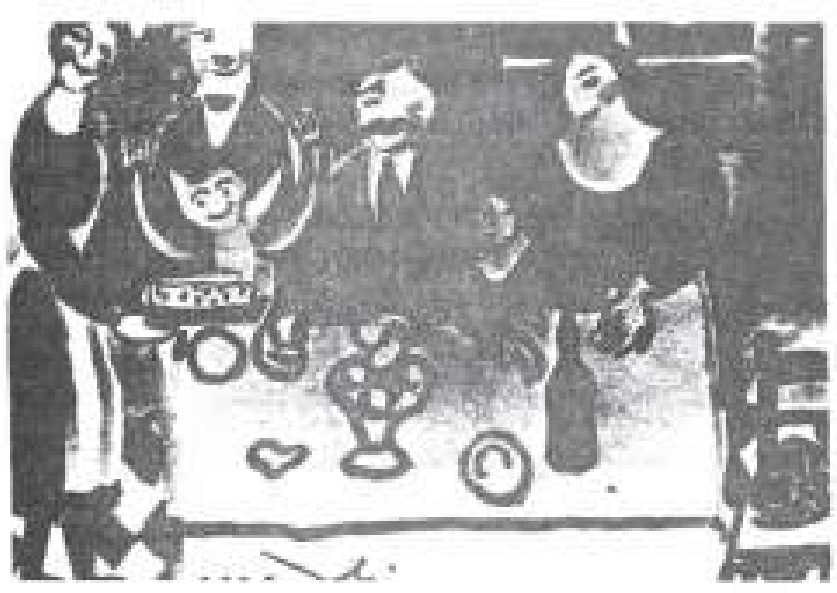

ORLANDO LONDONO BETANCOURT

Filósofo

Director Departamento de Ciencias Humanas

Universidad Autónoma de Manizales.

Profesor Universidad de Caldas.
STRUCTURING THE INDIVIDUAL THAT CONTEMPORARY SOCIETY NEEDS.THE MACROCURRICULUM AS A COMPLEX THINKING EXPERIENCE

$A^{\text {BSTRACT }}$

The Universidad Automoma de Manizales decided to redesign its curricular saructure as a product of its activities on strategic planning. This redesign resulted in the creation of a Macrocurriculum as an alternative leading to the holistic formation of its students, It consists of three areas: Sociohumanistic component, foreign language componemt and the component dealing with the development of managerial mentality. This Macrocurriculum is no doubt a great achievement of the Universify: esperially because it is a clear evidence of how education must be in the XXI century thus, being the macrocurriculum an institutional experience on complex thinking. This macrocurriculum is the main object of this paper. 


\section{RESUMEN}

La Universidad Autoinoma de Manizates, como producto del ejercicio de planeación estratégica, decidió reediseAlar la estructura curricular. Esa reforma llevo a que se creara como atternativa a la formación integrul de sus estudiantes un Macrocurriculo, el cual esta compuesto, en términos del plan de estudios por tres áreas asi: Componente sociobumanistico, componente de idiomas extranjeros y componente de desarnollo de la mentalidad empresarial. Este Macrocurriculo es sin duda un gran logro de la Universidad, sobre todo porque es una clara muestra de como debe ser la educación del sigio XXI. sobre todo, cuando el macrocurriculo es sin duda un experiencia institucional de pensamiento complejo. Este macrocurriculo es el objeto de este trabajo.

Los griegos habian comprendido muy bien qué significaba formar al hombre que necesitaba ia polis, es decir, entendieron que debía haber coherencia entre la educación, la cultura y la sociedad para la cual se educaba. En ese sentido. la forma de asumir el conocimiento en Grecia es esencialmente diferente a como lo asume la modernidad, pues, si bien es cierto que en ambos periodos de la historia el conocer se convertía en un elemento que se le exigia al hombre que se queria constituir en ciudadano y que, por tanto, va a la academia, al liceo, o los centros de formación universitarios modernos; tambièn es cierto, que ese saber, en el caso griego, estaba determinado por la necesidad de que el sujeto se formara de manera integral, esto es, que no sólo bastaba como sucede en la modernidad que el conocimiento esté mediado fundamentalmente por to racional, por aquello que el individuo podía explicar por medio de la razón. (lo cientifico); sino que también se le daban posibilidades al individuo de encontrar la integralidad del mundo desde la razón y desde otras esferas de lo humano. ${ }^{2}$ En este sentido, la explicación lógica del mundo que habia dejado Aristóteles y que habia perdurado durante 1700 años, todavía dejaba sus restos en el hombre moderno y en la forma en que éste asume el mundo. La ciencia moderna con-su fuerte rechazo a la física y a la cosmologia aristotélica, habia seyuido el rumbo trazado por su lógica, la cual nos da una sola posibilidad, además de unilateral, de entender el mundo. El Hombre ilustrado moderno, especialmente el del siglo XVIII, añoraba volver al ideal griego, a la poesia, la literatura, la mitologia, y la paideia, tal como lo muestran "las cartas para la educación estética del hombre" de Shiller. Grecia se convirtió en un ideal. Pues, allí hombre, educación, cultura, eran una misma cosa en tanto contribuian a la formación de una nueva polis. El ideal de educación griega. tal como lo muestra Jaeger, no es más que un hombre formado integralmente. El hombre moderno, tal como lo vernos al menos hasta hace muy pocos años, no era más que el ideal del sujeto ilustrado del siglo XVIII, un sujeto formado en la unilateralidad que da un conocimiento fraccionado, no pertinente, y alejado de lo que debe de ser la polis que se anhela tener.

Akira Okubo, investigador de la Universidad del estado de New York, y quien se ha coavertido en los últimos tiempos en uno de los científicos más importantes en ciencias biológicas, sobre todo por sus investigaciones en "Crecimiento de la organización biológica en ambientes turbulentos", en 


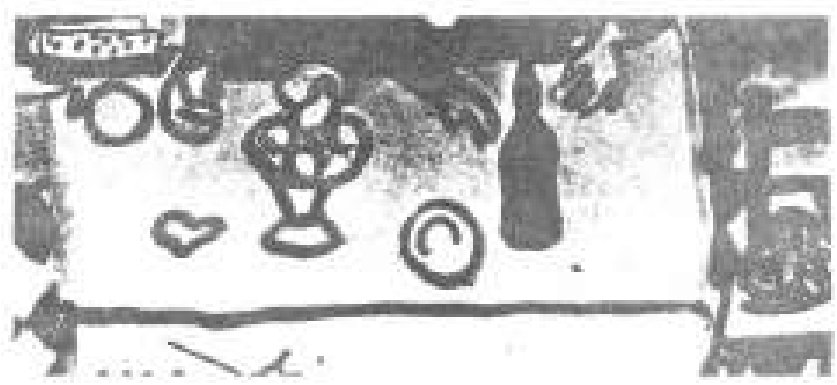

el "congreso internacional sobre imaginación científica" dijo refiriéndose a los errores en que cae la educación tradicional, y lo más paradójico del asunto es que lo hace colocando como ejemplo al Japón. Dice Okubo lo siguiente:

Lamento tener que decir que el sistema Japonés no hace demasiado favor del desaroollo de la imaginación. La primera es el aspecto tromerdamente competitivo qae hace que buena parte de la enseñanza este concentrada hacia la superación de los exdmenes (sobre todo los de ingreso a la universidad). El estudiante debe aprender todo to que el profesor le proponé. No queda espacio para aquello que liuego no va a hacer explicitamente exigido. En EE.UU, la enserianza es mais libre y se atiende a la opinion que puede tener el estadiante. El sistema puede tener tambièn sus defectos. pero al menos se favorece la comanicación no solo entre el profesor y el alamno sina también la comanicación entre alumnos. $Y$ La comunicación enseña a imaginar. He visto con frecuencia coimo estudiantes que hacen un mal examen. dan pruebas de una gran imaginaciain cientifica, $Y$ hay sistemas en los que ni siquiera es posible que se presente la oportunidad de que tal cesa pueda llegar a ser observada. Como japonés me dwele tener que reconocerlo.'

Más adelante, Carios Ulises Moulines, dice:
En este aspecto hay que comparar la imaginación cientifica con la imaginación artística. Lo que impulsa la imaginación en el cise o la literatura es el deseo de contarnos una historia coberente y plausible sobre el mundo. para lograr una unidad que no tie. ne nuestra experiencia inmediata. Esta sensación de unidad globales es lo que de hecho queda de una buena película o de una buena novela."

Lo planteado por Okubo es una represeatación clara de la forma como en los uítimos años ha sido entendida ta formación del individuo moderno (y sobre todo en el contexto de nuestro pais) Algo por su puesto muy diferente a lo que habia logrado Grecial, y a lo que recientemente se puede leer en el informe de la comisión internacional sobre la educación para el siglo $\mathrm{XX} 1$, precedida por Jacques Delors: o a la teoría de la complejidad, propuesta por el profesor Edgar Morín, y con base en la cual él escribe el documento, por encargo de la UNESCO, titulado ${ }^{*}$ Los siete saberes necesarios para ha educación del futuro"

El hombre contemporáneo es consciente de que la actitud moderna, ilustrada, frente al conocimiento ya no es la más apropiada, pues, el problema de cuánta información puede poseer una persona está determinado por ctuâ tecnologia puede utilizar, y no por cuánta capacidad de 
memoria tiene, es decir, la preguntas es por cuánta información puede guardar con la ayuda de la tecnologia. Lo anterior. supone entonces que una persona puede guardar mucha más información en un computador. Así, entonces, la cues. tión no estấ en qué tanta información poseer en nuestro pensamiento, sino en qué se va a hacer con ella, es decir, cómo utilizar, seleccionar, y enriquecerse con la información que se tiene.

Al respecto, Jacques Delors dice:

\begin{abstract}
-.tes que ya no tasia con que cadia individivo acumule al camienzo de su vida una reserva de conocimiento a la que podrá recurrir después sin limites. Sobre todo, debe estar en condiciones de aprovechar y urillzar durante toda la vida cada opurmuidad que se le presenre de acrualizar, profiundizar, enriquecer ese primer saber y de adaptarse a un mundo en permanente cambio?
\end{abstract}

Es por todo to anterior que también como lo plantea de Delors "...la educación se ve obligada a proporcionar las cartas náuticas de un mundo complejo y en perpetua agitación y. al mismo tiempo. la brújula para poder navegar por él": Era esta situación problemática la que llevaba a que la comisión planteara como pilares de la educación en el siglo XXI cuatro saberes. "Aprender a conocer". "aprender a hacer". "aprender a vivir juntos" y "aprender a hacer". Cada uno de ellos debe recibir una atención equivalente a fin de que la educación sea para el ser humano, en su calidad de persona y de miembro de la sociedad. una experiencia global y que dure toda la vida en los planos cognitivo y práctico?

A diferencia de Delors. Edgar Morín, ya no habla de 4 saberes, sino que propone como saberes fundamentales siete: "las cegueras del conocimiento". "Los Principios del conocimiento pertinente". "enseñar la condición humana", "enseñar la identidad terrenal". "afrontar las incertidumbres", "enseñar la comprensión", "la ética del genero humano". Staberes estos que debe. tal como lo dice el mismo autior, tratar toda sociedad y cualquicr cultura sin exclusión ni rechazo alguno, y que debe de eatenderse según las costumbres propias y las reglas propias de cada sociedad y de cada cultura.

Estamos por tanto convencidos que en un mundo Global, en el cual la reivindicación de lo cultural determina el que hacer del ser humano, en tanto en cada momento busca incesantemente su identidad. es necesario que propuestas como la de Detors o las de Morin sean asumidas con el interés de que ellas contribuyan, desde su ejecución, a mejorar el sistena educativo, a ampliar el horizonte cuitural del individuo, a lograr a través de la comprensión la posibilidad de la convivencia pacifica, a lograr mediante el ejemplo una apropiación por parte del hombre de sus valores, es decir, a que la persona se comprenda como individuo, complejo que es, en plena acción social, es decir, como un ser que está en compenetración constante con lo social. Un individuo que se entiende como ciudadano.

La reflexión debe ay udar a que el individuo entienda lo que significa el imperio del hombre en

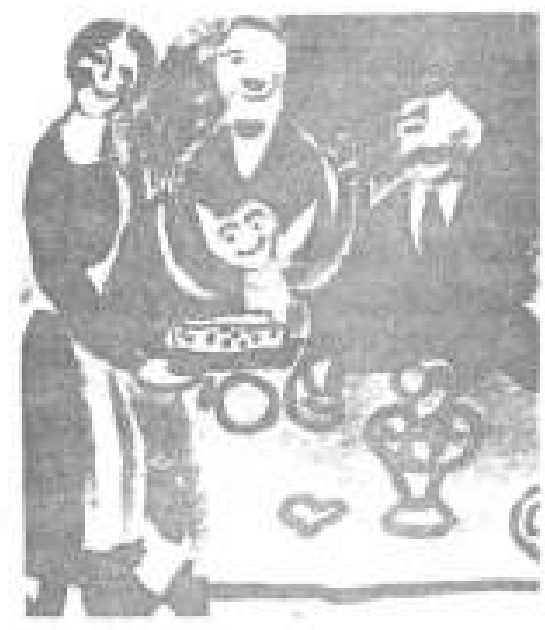




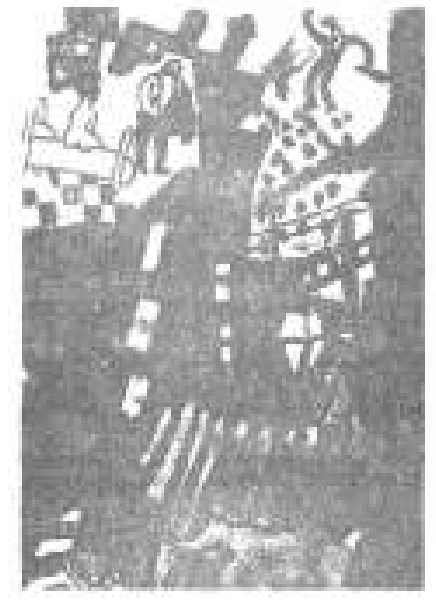

Ia tierra, y en ese sentido, logre comprender históricamente lo que él es, pero en relación con su entorno. Que entienda el verdadero significado de la vida; que promulgue la cuitura de la individualidad, pero con un profundo sentido de la solidaridad. Un individuo formado en el conocimiento de lo pertinente, pero con un desarroHo tal de las habilidades y destrezas de pensamiento, que le permitan entender la incertidumbre, lo azaroso, lo compiejo. Que comprenda las incertidumbres y las antinomias, que se desenvuelva en la multidimensionalidad. Es decir, que supere lo simplemente racional, y se ubique en lo emotivo, lo filosófico, lo antropológico y lo cultural, como condiciones necesarias para poder comprender ia complejidad de la vida. Todo lo anterior implica que el método, la didáctica y la forma de asumir el conocimiento, es decir, los contenidos deben cambiar.

En Colombia, aparecen propuestas similares, sobre todo cuando en el informe de la comisión sobre "Ciencia, Educación y Desarrollo" el científico colombiano Rodolfo Llinás decía, refiriéndose al reto de la educación lo siguiente:
La globalización determina huovas formas de interdependencia y jerarquias en fos sixtenas econdimicos y de ta information y el conocimiento mundiales, Estas desafian el pasado de muchas culturas, y les imponen a los valores culturales locales diferenciaciones econónicas y polaticas que los condicionan. Civilizaciones y naciones se confrontan actualmente a nivel mundial, en una competencia intelecrual que determina el acceso desigual a recurses, calidad de vida $y$ creatividad. Estas condiciones han gestado una mueva visión del mundo en las que los avarices de la ciencia y la lecnología, asi como los sistemas de ediacación y de organitación innovativas juegan el rol fundamental.

Las recientes crisis sociales y ambientales de los paises industrializados demuestran que la productividad economica y tos anamces del conocimiento humano requieren fundamentación en un conresto civilizador, cuyo fin ha de ser el bienestar social y el respeto por la vida. En consercuencia, el ilesarrollo, entendido cramo el atuance humano, económico, politico y cuitural, debe construirse como un legado de información al servicio de estilos de villa inseligentes y garantes de la creatividad humana para fu. turas generacianes.

Lo anterior reguiere una restructuración y rewolución de la edtacicioin, que genera et nuevo ethos cuitural, que potencie al maximo las capacidades intelectuales y organizativas de los colombianos. Una manera innowativa de entender y actuar - no el simple saber y hacer - debe permitir que se adquieran nuevas habilidades humanas. basadas en el desarrollo de multiples saberes y talentos. tanto cientificos come artisticos y literarios y debe servir para gestar nuevas formas de organización productiva."

Es así como la Universidad A utónoma de Manizales comprendió que el que bacer educativo debia implicar muchas otras cosas, cosas que debian tras. cender el simple hecho de formar profesionales. 
La Universidad, en general, es para la sociedad colombiana de hoy, un antivalor, pues, para vivir en Colombia, el mundo del conocimiento es. sin duda, contradictorio; sobre todo si se piensa en una sociedad que es corrupta, antiética, poco competitiva y generadora de violencia. La universidad forma hombres para que logren todo lo contrario, y es en ese seatido que creemos que la misión de la universidad debe de estar com. prometida con la formación de una nueva "polis" en el sentido literal griego. Es decir, en la construcción de una nueva sociedad. La universidad no sólo debe preparar individuos para que estén en condiciones de adaptarse al mundo de hoy. sino que además, para que en prospectiva puedan hacer, maniana, un nuevo mundo, O sea. personas que no sólo se adapten sino que también estén en condiciones de transformar.

Es fundamentalmente en eso que plantean los texios del profesor Morin. y en to que dice ol Dr. Llinas en to que se ha convertido el acto educativo para la Universidad Autónoma de Manizales, en todo un reto: convertirse en una institución que a través de la formación rigurosa de sus alumnos pueda responder a los problemas que le genera el medio social que lo rodea, esto es, que nos hemos propuesto formar individuos, tanto profesional como humanamente competentes para la solución de problemas reales: lo anterior, implica ver la educación superior más que como una herramienta, como la máxima posibilidad de desarrollo que tiene la región.

En pro de cumplir con tal cometido, aparece como estrategia, lo que podriamos llamar una verdadera experiencia de pensamiento comple- jo, es decir, una experiencia educativa que conlleva a cumplir en gran medida con to que el protesor Morín propone, y a desarrollar pensamiento complejo desde la perspectiva de la didáctica, la pedagogia, y la forma de abordar el conocimiento. Esta experiencia la hemos denominado Macrocurriculo.

Pretendemos, por tanto, que el macrocurriculo permita constituir la enseñanza no sólo como un acto que forma profesionales, sino también como formación integral del individuo. Formación que responda a la comprensiōn del medio a partir de la reflexión y de la ircción sobre él. Una formación que responda a la posibilidad de fundamentar esencialmente a cada uno de los saberes; que sea humanista por excelencia, esto es, que forme personas refiexivas, crificas, dinámicas, y conscientes de la necesidad de pensar en la realidad que se vive. Para que así se constituyan en sujetos históricos que hagan el reino del hombre aquí en In tierra:

Es cierto que si queremos responder a los intereses de is sociedad de hoy, podríamos decir que con ta mera formación en la profesión basta, pero ¿hasta adonde a esos profesionales tes es suficiente, en cuestión de calidad, competitividad, y de la posibilidad de ubicar históricamente su quehacer, la sola formación profesionalizante?. La profesion es importante en la medida en que vaya acompañada de unia preparación para la vida, con le cual el estudiante esté en condiciones históricas, sociales y políticas de dar cuenta no sólo de su realidad, sino también del papel que juega la profesión que eligió, en el contexto de la realidad en la cual vive. En este caso, la educación 
debe partir de la pregunta ¿Qué persona queremos formar? y ¿Qué características debe tener esa persona que es formada en nuestro sistema? La primera pregunta no se puede responder. como tampoco la segunda desde un solo plano, sino que su respuesta ya es en sí misma compleja, Es decir, que la respuesta conduce a la pregunta ¿qué tipo de sociedad queremos formar. para cuál sociedad se quiere formar, qué características tiene esa sociedad?. Preguntas estas que sin duda se hacian los griegos cuando de educación se trataba, pues, ello implicaba responder por una nueva polis, por la nueva sociedad que se quiere construir. Pero la complejidad de la preguntá ya mucho más allá, cuando en medio de todas las respuestas nace la pregunta, ¿cuál es el conocimiento apropiado o pertinente para formar esa persona, con las características que tiene $\mathrm{y}$ debe tener la polis para la cual lo preparamos?. Pero, ¿cómo se logra tał cometido?, ¿cómo se debe concebir la universidad para lograr tal propósito? ¿de qué supuestos teóricos debe partir entonces la educación universitaria?. La respuesta es compleja, pero determinante para poder entender en su contexto lo que hemos querido hacer.

Responder esto implica, entonces, hacer una mirada antropológica, sociológica, económica y política del problema educativo universitario. El individuo aparece como factor de construcción esencial en una sociedad, y por ello se hace necesario pensarlo, desde su formación, como el factor esencial del cambio hacia una nueva época. Todo lo anterior, supone que se conciba, independiente de la profesión un individuo integral, interdisciplinario, creativo, y con un amplio desa- rrollo de la imaginación. Es decir, un ser consciente de su relación eomo individuo con la sociedad y la especie, además del conocimiento.

Las grandes escuelas se caracterizan porque ven más allá de la inmediatez que le proporciona su existencia, pues, esta inmediatez conduce a una aprehensión de la realidad que genera una subvaloración errada de todo aquello que intetroga por lo que hay al interior de lo que cientificamente es la profesión y de lo que ella significa para la sociedad. Es por ello que el estudio de las áreas del conocimiento que se preocupan por la formación de carácter generai permite, alejándose de la inmediatez. lograr con mayor eficacia los procesos interdisciplinarios tan necesarios hoy en la investigación cientifica, que como se ha dicho es en últimas junto con la docencia y la proyección. el pilar que mueve la universidad.

El profesional que queremos format con el macrocurriculo, tiene que saber cual es el mundo que habita, lo cual se logra entendiéndolo como mundo físico, y al mismo tiempo. debe comprenderlo como un mundo social, político, económico: para el cual su profesion lo ha preparado desde todos los punto de vista.

Se necesita un hombre competitivo, integral, formado no sollo para ser el mejor profesional, sino para ser la mejor persona. Un individuo consciente de la realidad en la que vive, critico frente a ella, pero con ánimo de trasformarla, Un ser humano consciente de la importancia del otro y, por tunto, que el otro se sienta validado por él independiente de sus diferencias, Un hombre con conciencia histórica. Con habilidades y destre- 


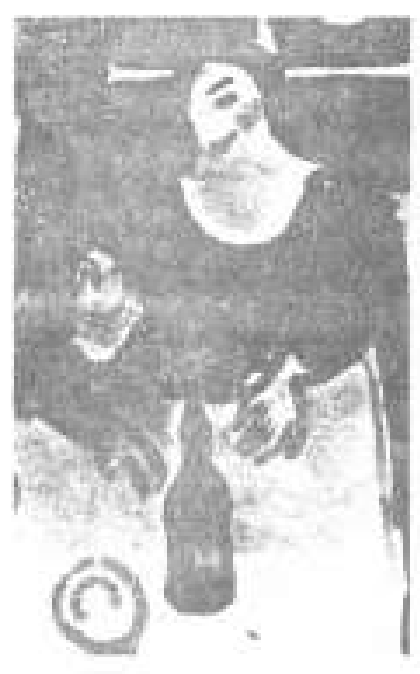

zas de pensamiento que le permitan resolver problemas y construir conocimiento. Una persona capaz de afrontar con calidad los problemas que el medio le genere, individuos lectoescrituralmente bien preparados, reflexivos, con el pensamiento lógicamente bien construido, matemáticamente competentes, pero ante todo humanos. conscientes de cuál debe ser su acción en el mundo.

A través de eși acción la persona debe estar en capacidad de conocerse a sí misma, es decir, de entenderse como un hombre en unión intersubjetiva con el mundo y no aislado de él como simple observador, o que en el proceso de conocimiento de si mismo tenga que abandonar el mundo e interiorizarse de tal manera que el mundo desaparezea frente a sus ojos. La persona que se piensa es fundamentalmente un ser que tiene el mundo a la mano, es decir, que el mundo, la realidad, el otro hinga parte de su conjunto de preocupaciones.

Debemos de reconocer que ya no vivimos en una sociedad tribal. mágica, en la que no se distingue la dualidad de hechos y normas, en la que no hay libertad ni democracia. Por el contrario. hemos reflexionado sobre una sociedad diferente, la cual se basa en el dualismo entre las nor- mas y los hechos y en los valores de libertad, igualdad, humanidad y razonabilidad. Es decir. una sociedad que fomente ef respeto por los valores que el liberalismo y democracia trajeron consigo: libertad e igualdad.

Así mismo, Vivimos en una época en la que la teoria, que se refiere al ser inmodificable de las cosas que se encuentran más allá del dominio cambiante de fos actos humanos. sólo gana una vigencia en la praxis en la medida en que ella misma logra acuñar la actitud vital del hombre que se ocupa con la teoria, en que les abre también normas, a partir de una comprensión del cosmos en su totalidad, para el propio comportamiento, y en la medida en que toma una figura positiva a través de las actuaciones de aquellos que tiene una formación integral, y no solo en lo competente a la respectivat profesión.

Este macrocurriculo está dividido en tres partes bien definidas, Estas son: formación sociohumanística: (la cual a su vez se divide en dos, La formación en habilidades y destrezas de pensamiento y la formación sociohumanistica propiamente dicha). Formación en idioma extranjero; y formación en desarrollo de la capacidad empresarial. Cada una de estas áreas está curricularmente representada por asignaturas que hacen parte del curriculo de cada uno de los programas académicos y que deben cursar obligatoriamente todos los estudiantes de la Universidad.

El macrocurriculo que estamos presentando aquí tiene como objetivo cumplit con la serie de saberes propuestos por Morin, esto es, que al alumno se le forma en diferentes áreas, concibiendo de manera integral el conocimiento. Así, desde la enseñanza de la lectoescritura, de la construcción det pensamiento, de la fillosofia de la ciencia, se conduce al desarrollo de habilidades y destrezas de pensamiento. Tales asignaturas, producto de la forma como se transmiten, y 
de los contenidos, cada vez más ajustados a lo que es conocimiento pertinente, han permitido que el alumno logre alcanzar niveles de pensamiento superiores a los tradicionales, en tanto la visión del todo, de su propia profesión y de la vida en sociedad sun comprendidas y asimiladas como un sistema complejo.

La ensenanza de la filosofia de la ciencia, por ejemplo, está diseñada de tal manera que el estudiante pueda comprender, desde la mirada de las diferentes teorias epistemológicas, lo que hay de cientifico en su profesión, es decir, el estudiante a través de la resolución de problemas logra diferenciar entre las teorias y el conjunto de aplicaciones que tiene la profesión, cuestión ésta que instała al alumno en una lógica diferente a la tradicional, en tanto le muestra el problema de lo teórico bajo relaciones diferentes. es decir. mediante relaciones sistemáticas de teorías. El estudiante se da cuenti que el todo no es efectivamente la suma de las partes, pues, no se puede olvidar de considerar la estructura que damos a las partes para formar el todo. Asi, un sistema está formado por elementos que interactúan, pero no están relacionados unos con otros de manera totalmente rigida, sino flexible. admitiendo cierta indeterminación, introducida en gran parte porque los sistemas se extienden en un espacio.

La biologia, por ejemplo para el caso de los programas de la salud (odontología y fisioterapia). acepta muchas veces la física como punto de partida. Los sistemas fundamentales de la física (átomos, partículas elementales, etc.) se toman adecuadamente como material para describir la vida. Pero el mundo de la vida en continuidad con el mundo de la física no es más que un problema, me parece a mi, de información y organización. Se podría llegar a un resultado parecido sustituyendo ese material por otro 0 , simplemente, sustituyéndolo por simples símbolos o piezas de construcción de una especie de super-

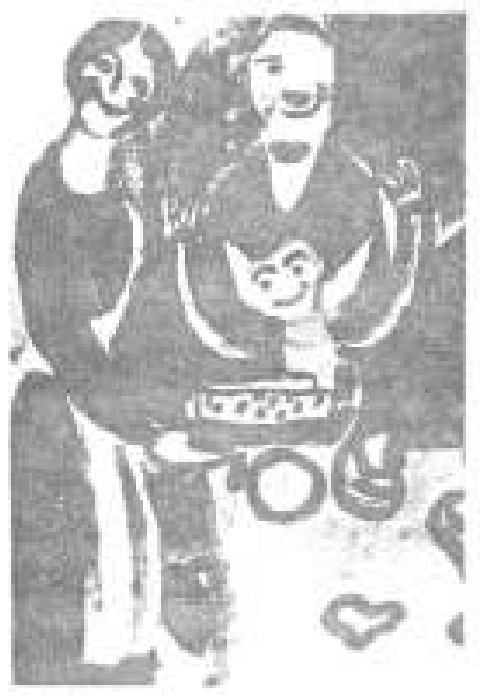

estructura. Se ha manifestado la sospecha de que la física podria quedarse pronto sin asuntos fundamentales que estudiar. En biología, en cambio, quedan aum muchas cosas que no entendemos. pero todas csas cosas tienen siempre relación con tał superestructura. La célula, por ejemplo, no es un caso de enzimas, y su peculiar y precisa organización todavia se nos escapa en gran parte. El desarrollo del organisino tampoco está claro en muchos aspectos. Poca cosa sabemos de la memoria que usamos cuda día. Aún queda muchat cosa por investigar. Pero, en las listas de cosas fundamentales de la ciencia aún pendientes no suclen aparecer todavia el aumento de la complejidad, la memoria. la organización, la información. Eso creo, tiene una alta relación con la fisica, pues, el funcionamiento de sistemas que no están totalmente conectados entre sí viene dado simplemente por la cuantificación, esa tendencia de la vida a formar unidades discontinuas. La biología tiene mucho trabajo por hacer, pero gran parte de ese trabajo es compartir con otros dominios dedicados a sistemas no vivos de distinto grado de organización, en los que también se producen intercambios de información."

El ejemplo anterior acerca de la enseñanza de la filosofia de la ciencia, no es mas que una parte 
de cómo se está aplicando el conocimiento pertinente, y qué producto del mismo está dando posibilidad a la incertidumbre, a lo mítico, pues. en este último caso no se puede negar la relación que existe entre mito y ciencia. Ambas responden al deseo de comprender el mundo. Pero puede existir una gran diferencia entre el hecho de la simple y pasiva aceptación de los mitos y la activa investigación y persecución de la comprensión del mundo. El conflicto de creer profundamente en una verdad con la comprobación de que ésta es insostenible. Es de hecho el conflicto entre el deseo de crear y lo que podriamos llamar la honestidad intelectual.

La cátedra de lectoescritura, tal como la de construcción del pensamiento, o las del componente de desarrolio de la habilidad empresarial, permiten el desarrollo de las habilidades y destrezas de pensamiento para que el individuo comprenda, se comprenda y explique el mundo. Es decir, son aquellas asignaturas que permiten al igual que las demás el encuentro interdisciplinario entre los diferentes saberes. con la diferencia que aquí los encuentros están mediados por el desarrollo de habilidades, sobre todo dirigidas a la resolución de problemas, (distinguir entre oportunidades y riesgos), a la comprensión del mundo como un todo, $y$ a pensar con una lógica, más que formal, concebida de manera diferente para resignificar el mundo. La complejidad.

Por otro lado el sujeto es formado en saberes humanistas como la socioantropología, la ética y la cultura política. Aquí, el saber supera los límites de la profesión, y se encarna en la vida misma. El individuo se forma como ser ético. político, democrático, respetuoso de las diferencias, comprometido con la convivencia pacifi- ca. La cátedra de socioantropología, por ejemplo, permite que el hombre se entienda asi mismo, pero no aislado del entorno. Es aquí donde la categoría "acción" cobra gran significación. en cuanto el individuo no es posible en el solipsismo, sino en su conexión con el otro y con el mundo.

En definitiva, el macrocurriculo es una experiencia naciente de lo que es el nuevo paradigma de la complejidad. Es el encuentro del texto sobre los siete saberes del profesor Morin. y una experiencia en la que cada saber está siendo posible, en tanto hay un curriculo creado para ello.

La reforma curricular creó el mucrocurriculo para que respondiera a la formación del profesional que necesita el país, fo que es aquel en la actualidad, responde a tai cometido, además de contribuir de manera Holista por una forma integral de ser del hombre y del conocimiento. La sociedad y el mundo globaies.

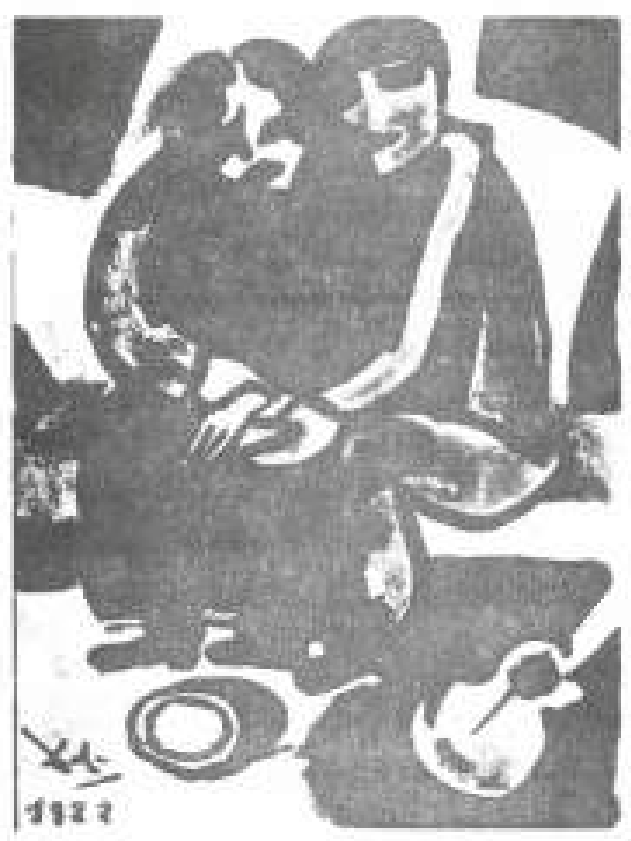




\section{NOTAS}

\section{BIBLIOGRAFIA}

'A.SEN y M.C. Nussbaum. compilaciones. Caidad de vida. F.C.E. 1996

* Una cuestión interesante al respecto es la exigencia que te hacia de que el hombre aprendiera matemáticas, muisica, geometria, es decir, que se formara no sólo en lo que competia at su campo de acción. sino que también estuviera en capacidad. de conocer el mundo ea su más alta complejidad, ello explica el por qué la cultura helénica es esencialmente humanista.

" OKUBO, Akira. Intervención en el debate. En. HAKEN. Hermann, et al. Sobre la imaginación Cieatifica. Edición de Jorge Wagensberg. Traducción de J M. Llosa. Barceiona. Tusquets. 1990. Pp 116.

+1BID. pp 117.

'DELORS. Jacques, La educación encierna un tesang. (informe a la UNESCO de la comisión internacional sobre la educacioin para el siglo XXI). Madrid. Santillana. 1996. Pp 45.

*IBID. Pp 95

IBID. Pp 96.

" Ver; ALDANA VALDEZ, Eduardo el al. Colombia al filo de fa oporranidad (mision ciencia. educación y desarrollo). Santafé de Bogotá D.C. Tereer mundo Editores. 1996. pp 62 63.

* La ciencia es un sastema de información que se adquere. según Nikłas Luhman, mediante un metodo de analisis funcional, este método, de orden teórico, es una modalidad especifiea del mundo de la vida, que apenta a caplar la diferencia de los saberes, sus relaciones, los istereses del conocimiento, la complejidad, la contingencia. Para ello se sirve de la comparación. Es la comparación lo que permite "compreader lo existente como contingeate, y lo distinto coms comparable".
A.SEN y M.C. Nussburm, compliaciones. Calidad de vida. FC.E. 1996

DEL.ORS, Jacques. I a edacación encierra un iesoro.(iaforme a la UNESCO de la comisión internacional robre la educacioin para el siglo XXI). Madrid. Santiflana. 1996. Pp 95

HAKEN, Hermann, et al. Sobre la imeginación Clentifica. Edicioin de Jarge Wagensherg. Tnducción de J M. IJom. Barcelona. Tusquets. 1990. Pp 116

MORIN, Edgar Iniroducciùn al pensamaienio complejo. Traducsión de Marcelo Pakinan. Burcolona, Gedisa. 1996. Ppl67.

Los siele saberes accesarios para la educación del futura. Traducciós de Mercedes Valicjo - Goumez. Santafé de Bogotá. 1999.pp 90

EL Mirode. (la naturaleser de la naturaleza). Cuarta edición. Traduccion de -Aru Sanchez y Doca Sanchez

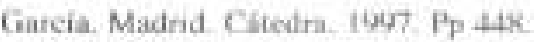

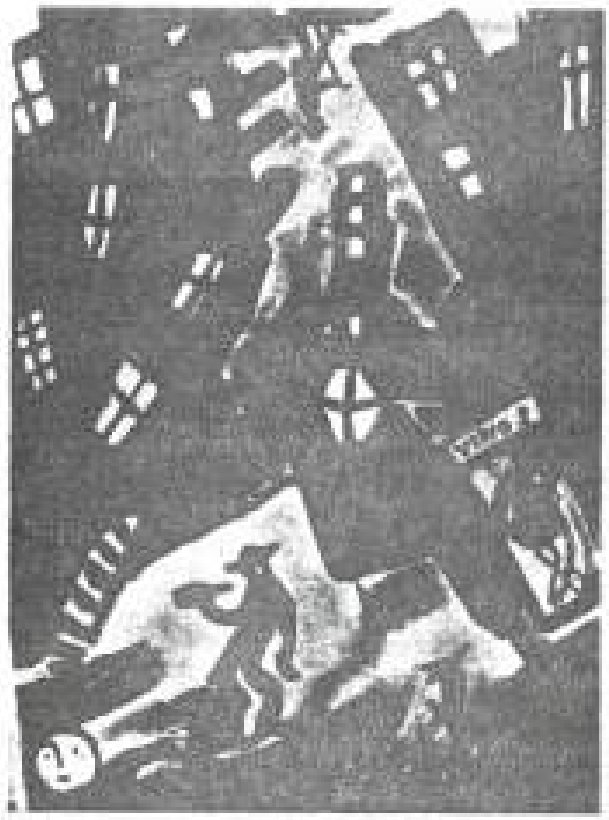

